\title{
Treatment with a recombinant human IgM that recognizes PSA-NCAM preserves brain pathology in MOG-induced experimental autoimmune encephalomyelitis
}

\author{
Hernan Nicolas Lemus ${ }^{\mathrm{a}}$, Arthur E. Warrington ${ }^{\mathrm{a}, *}$, Aleksandar Denic ${ }^{\mathrm{a}}$, Bharath Wootla ${ }^{\mathrm{a}}$ and \\ Moses Rodriguez ${ }^{\mathrm{a}, \mathrm{b}}$ \\ ${ }^{a}$ Department of Neurology, Mayo Clinic, Rochester, MN 55905, USA \\ ${ }^{\mathrm{b}}$ Department of Immunology, Mayo Clinic, Rochester, MN 55905, USA
}

\begin{abstract}
A single peripheral dose of CNS-binding IgMs promote remyelination and preserve axons in a number of animal models of neurologic disease. A myelin-binding recombinant human IgM (rHIgM22) is presently in a safety trial in MS patients following an acute MS exacerbation. rHIgM22 (directed against oligodendrocytes) or rHIgM12 (directed against neurons) were administered to mice with MOG-induced experimental autoimmune encephalomyelitis (EAE) with study endpoints: clinical deficits and brain and spinal cord pathology. IgMs were administered at a therapeutic dose of $100 \mu \mathrm{g}$ intra peritoneal at the time of immunization (day $-1,0,+1$ ), disease onset (15 days) or peak of the disease (28 days). Disease course was not worsened by either human IgM regardless of the time of treatment. Of note, the human IgM that recognizes a carbohydrate epitope on gangliosides and NCAM, rHIgM12, reduced brain pathology when given at time of immunization or at onset of disease, but did not reduce clinical deficits or spinal cord disease burden. Hence, treatment with rHIgM12 resulted in marked reduction in meningeal inflammation. Data consistent with the hypothesis that in the EAE model this molecule has an immune-modulatory effect. Treatment with an anti-CD4 blocking IgG prevented both clinical course and CNS pathology. This pre-clinical study further supports the safety of therapeutic CNS-binding human IgMs in the presence of autoimmunity and clearly differentiates them from IgGs directed against MOG or aquaporin-4 that worsen neurologic disease.
\end{abstract}

Keywords: Human antibody, experimental autoimmune encephalomyelitis, myelin oligodendrocyte glycoprotein, inflammation

\section{Introduction}

Human antibodies are a potential novel class of therapeutic agents for multiple sclerosis (MS). Currently, natalizumab and alemtuzumab are the only monoclonal antibodies approved by the FDA for the treatment of MS. Still, there is no cure for the disease and many of these agents can only be used for limited time.

\footnotetext{
${ }^{*}$ Corresponding author: Arthur E. Warrington, Department of Neurology, Mayo Clinic, 200 First St. SW, Rochester, MN 55905, USA. Tel.: +1 507284 4663; Fax: +1 507284 1086; E-mail: warrington.arthur@mayo.edu.
}

There is an immediate need for new treatments for MS and other neurodegenerative diseases. Demyelinating diseases like MS would benefit from treatments that actively induce brain lesion repair and stimulate oligodendrocyte remyelination.

Murine models of MS are used by many laboratories to develop new remyelinating agents in MS [1]. The most commonly studied animal model of MS is experimental autoimmune encephalomyelitis (EAE) [2]. By immunizing with central nervous system (CNS) tissue or its components, such myelin basic protein (MBP), myelin oligodendrocyte protein (MOG), proteolipid protein (PLP) or adoptive transfer of CNSspecific $\mathrm{T}$ cells, an inflammatory cascade is initiated

ISSN 1093-2607/16/17/\$35.00 (C) 2016/2017 - IOS Press and the authors. All rights reserved

This article is published online with Open Access and distributed under the terms of the Creative Commons Attribution Non-Commercial License (CC-BY-NC 4.0). 
resulting in immune cell infiltration of the brain and spinal cord, demyelination and axonal loss. Some aspects of EAE resemble the complexity of MS in humans. Hence, EAE helps to understand the immunologic mechanisms involved in the pathogenesis of MS and to test a variety of therapeutic agents $[3,4]$.

Natural human antibodies are molecules present in newborns without stimulation by foreign antigens (i.e. part of the human innate immunoglobulin repertoire) [5-7]. Our laboratory has been investigating the potential of natural human antibodies for CNS repair. A recombinant human immunoglobulin M (rHIgM22) binds to myelin and stimulates remyelination in Theiler's murine encephalomyelitis virus (TMEV) infected animals [8] and accelerates remyelination in lysolecithin induced demyelination [9]. rHIgM22 also preserves the absolute number of spinal cord axons and their functional competency [10]. A neuron-binding, recombinant human immunoglobulin $\mathrm{M}$ (rHIgM12), promotes neurite outgrowth and preserves motor deficits in a chronic inflammatory demyelinating murine model [11]. rHIgM12 binds to the surface of neurons, oligodendrocyte progenitors and a population of astrocytes by recognizing polysialic acid (PSA) attached to neural cell adhesion molecule (NCAM) and to gangliosides GD1a and GT1b $[11,12]$.

Although therapeutic in the TMEV, lysolecithin and cuprizone models, the recognition of CNS could initiate an inflammatory cascade as a possible reactivity against different myelin antigens or neuronal structures. There is strong evidence that antibodydependent mechanisms contribute to the pathogenesis of CNS inflammatory demyelination [13]. Antibodies against the extracellular domain of MOG, MBP, PLP or aquaporin-4 (AQP4) are present in diseases such as MS and neuromyelitis optica. This is evidence that myelin can be a target of humoral immune reactions [14-19].

Based on this evidence we tested whether mice treated with either rHIgM22 or rHIgM12 exacerbate the clinical deficits or the inflammation and demyelination, in the CNS of a murine model of EAE induced by MOG. The study was not designed to investigate whether these agents induced remyelination or CNS repair in the EAE model.

\section{Materials and methods}

\subsection{Human recombinant antibodies}

rHIgM22 and rHIgM12 are recombinant human IgMs generated by expression cell lines constructed by our team $[20,21]$ and purified to greater than $97 \%$ purity using a 3 step column procedure, CHT Ceramic Hydroxyapatite, Type II (Bio-Rad), a CIM QA-8f Monolithic column (BIA Separations) and a Sephacryl S-300 HR column [10]. The final IgMs were validated using titrated potency assays of binding to the surface of live neurons, oligodendrocytes and tissue slices or the support of neurite extension.

\subsection{Disease induction}

Female C57BL/6 mice of ages 10 to 12 -weeks were anesthetized by inhalation of $4 \%$ isoflurane and immunized subcutaneously (s.c.) on the upper and lower back with MOG 35-55 peptide emulsified in complete Freund's Adjuvant (Hooke Labs Inc \#EK-2110). Pertussis toxin was injected intraperitoneally $2 \mathrm{hrs}$ and $24 \mathrm{hrs}$ after immunization.

\subsection{Clinical deficit scores}

Mice were observed daily for clinical symptoms and disease severity and scored as follows: 0 , normal; 1 , loss of tail tone; 2 , hind limb weakness; 3 , hind limb paralysis; 4 , hind limb paralysis and forelimb paralysis or weakness; and 5, moribund/death. Grading of mice was performed by two investigators blinded to treatment.

\subsection{Treatment with antibodies}

Mice were treated with a single $100 \mu \mathrm{g}$ i.p. dose of rHIgM22 or rHIgM12. Phosphate buffered saline was used as the vehicle control. GK1.5 (anti-CD4 IgG1, purified from cell culture supernatant) is known to suppress EAE inflammatory responses and was used as a positive control. IgM treatment was tested at three different time points of disease: Experiment \#1: treatment at 15 days post immunization (time when more than $50 \%$ of mice reached a clinical score of at least 1 ), Experiment \#2: treatment at 28 days post immunization (the peak of disease) and Experiment \#3: treatment with 3 separate $100 \mu \mathrm{g}$ doses of antibodies on days $-1 / 0 /+1$ ( 1 day before/at the time of/and 1 day post immunization). Therefore mice in Experiment \#3 received a total of $300 \mu \mathrm{g}$ of antibodies.

\subsection{Assessment of spinal cord pathology}

Mice treated in experiments \#1, \#2 and \#3 were anesthetized with sodium pentobarbital and perfused in- 


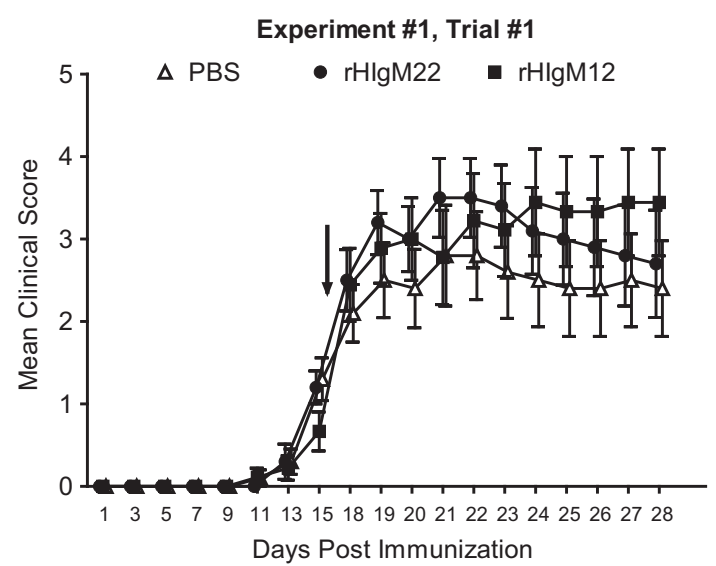

A

Experiment \#2

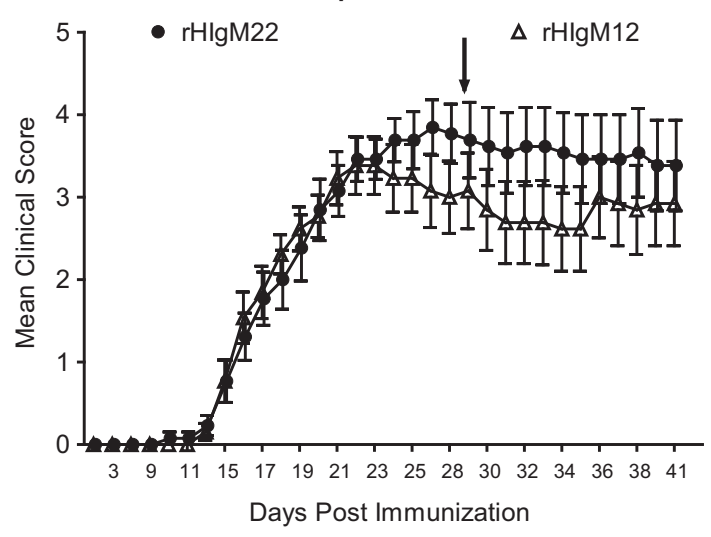

$\mathrm{C}$

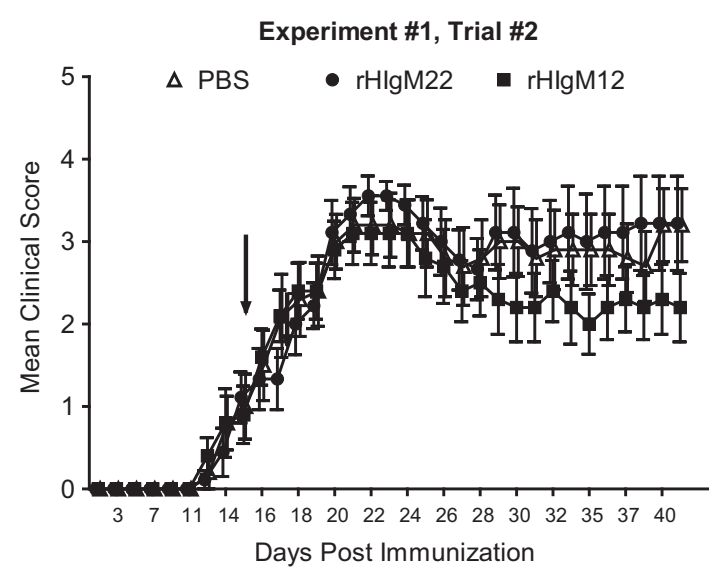

B

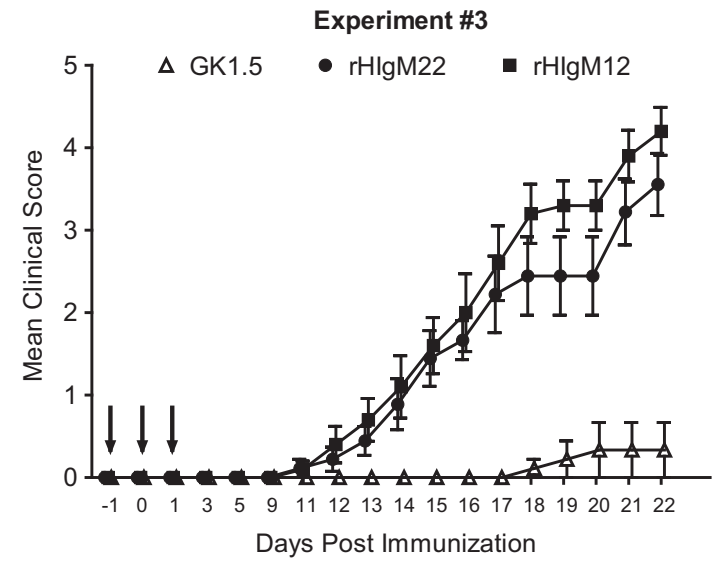

D

Fig. 1. The recombinant human IgMs, rHIgM22 or rHIgM12, did not exacerbate EAE clinical scores when administered at the onset of symptoms, the peak of disease, or immunization. C57BL/6 mice with MOG-induced EAE were given a single $100 \mu \mathrm{g}$ i.p. dose of rHIgM12, rHIgM22 or saline at different time points of the disease (black arrow in each graph): Experiment 1: treatment was given at 15 days post immunization; Experiment 2: treatment was given at 28 days post immunization; Experiment 3: treatment was given at 1 day before, at the time and 1 day post immunization. A: Mice were monitored and scored daily for clinical deficits out to 28 days post immunization. B: In a repeat study mice were monitored and scored daily out to 41 days post-immunization or until mice were moribund to assess whether potential remission was altered. C: C57BL/6 mice with MOG-induced EAE were given a single $100 \mu \mathrm{g}$ i.p. dose of rHIgM12 or rHIgM22 at day 28 post-immunization, typically the peak of disease in this model, and monitored until 41 days post-immunization. D. C57BL/6 mice were given three $100 \mu \mathrm{g}$ i.p. doses of rHIgM22, rHIgM12 or the anti-mouse CD4 IgG, at the time of immunization. Mice were monitored for clinical deficits until the typical peak of disease, 22 days post immunization. Only one mouse in the anti-CD4 treatment group developed minimal deficits late in disease, whereas 9 mice remained deficit free. For each study the daily mean disease score for each group was plotted. Error bars represented the standard error of mean. Rank sum tests of daily clinical scores did not reveal any significant difference between rHIgM12, rHIgM22 or PSB treated groups. The anti-CD4 treated group in panel D was significantly different when compared to the mice treated with rHIgM22 or rHIgM12.

tracardially with Trump's fixative (phosphate-buffered $4 \%$ formaldehyde/1\% glutaraldehyde, $\mathrm{pH} 7.4$ ). Spinal cords were removed and sectioned precisely into $1 \mathrm{~mm}$ blocks. In order to sample along the length of the spinal cord, every third block was post fixed, stained with osmium tetroxide and embedded in araldite plastic (Polysciences, Warrington, PA). One-micron sections were cut and stained with $4 \%$ p-phenylenediamine to visualize the myelin sheaths. Ten spinal cord cross sections, spanning the entire spinal cord from cervical to the dis- tal lumbar spinal column regions, were examined from each mouse. Each quadrant from every coronal section from each mouse was graded for presence of demyelination and inflammation. Areas of demyelination are characterized by naked axons, cellular infiltration and macrophages with engulfed myelin debris. Quadrants with inflammation are characterized by the presence of cells within the meninges or vasculature. Demyelination and inflammation scores were expressed as the percentage of spinal cord quadrants examined with the 


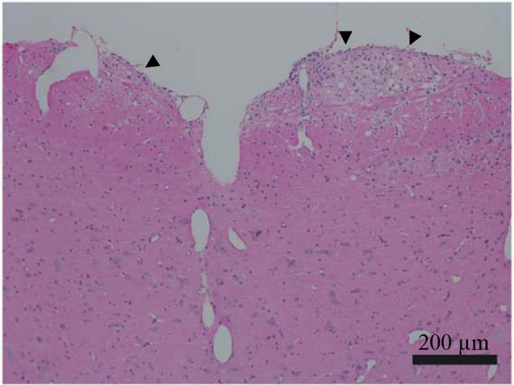

A

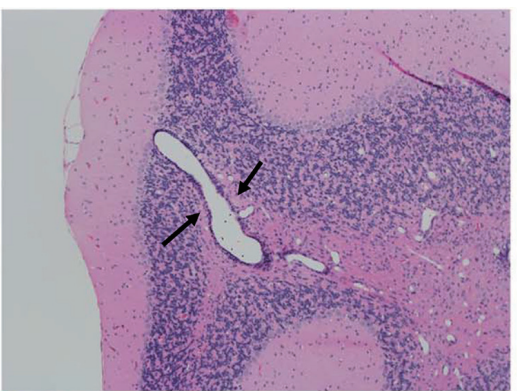

$\mathrm{C}$

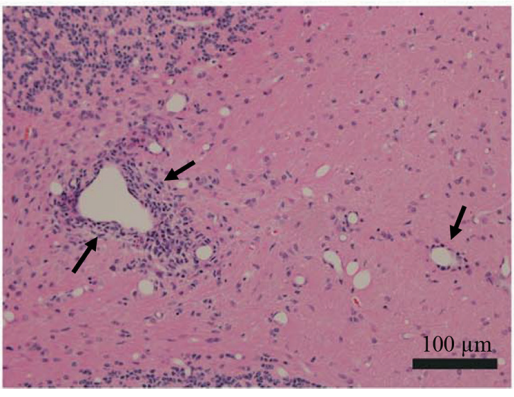

E
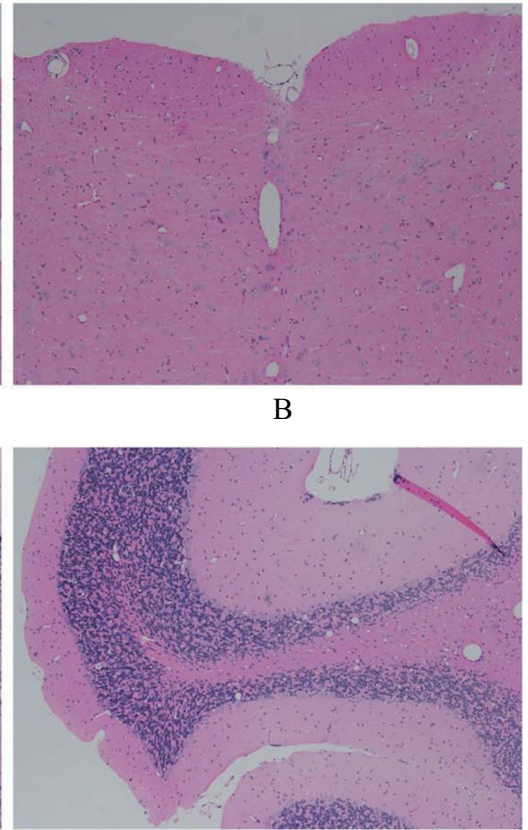

$\mathrm{D}$

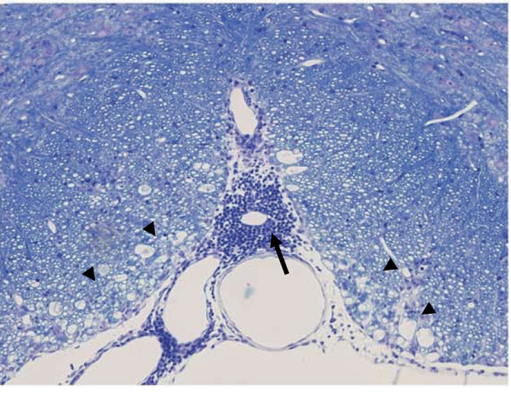

F

Fig. 2. Assessment of brain and spinal cord pathology. Seven areas of the brain were assessed for inflammation and tissue damage in hematoxylin and eosin stained paraffin cross sections. Ten eriochrome stained cross sections encompassing the entire length of spinal cord were scored for inflammation and tissue damage. The examples here are from experiment \#1, trial \#1. Brain stem tissue damage (demyelination) and immune cell infiltration (arrowheads) were present in PBS treated animals (A), whereas limited tissue disruption was found in rHIgM12 treated animals (B). Inflammation in the cerebellum was equivalent across all treatment groups. Typical perivascular immune cells within the cerebellar white matter and granular layer (arrows) are shown in (C) compared to normal folia structure in (D). An example of severe perivascular inflammation (4 or more layers, left side arrows in E) compared to more subtle single layer perivascular inflammation (right side arrow in E). Spinal cord cross sections were visually graded as quadrants for tissue damage and demyelination, and meningeal inflammation. Tissue destruction (arrowheads in F) and prominent meningeal inflammation (arrow in F) are present in both quadrants of this section of ventral spinal cord. $200 \mu \mathrm{m}$ scale bar in A applies to A-D, $100 \mu \mathrm{m}$ scale bar in E applies to E and F.

pathological abnormality. A maximum score of 100 indicated a particular pathological abnormality in every quadrant of all spinal cord sections of a given mouse. All grading was performed on coded sections without knowledge of the experimental group.

\subsection{Assessment of brain pathology}

Brain pathology of mice was assessed following perfusion (as described in the previous section). Two coro- nal cuts in the intact brain (one section through the optic chiasm and a second section through the infundibulum) resulted in three paraffin-embedded blocks. Five micron sections from these blocks stained with hematoxylin and eosin allowed the analysis of the cortex, corpus callosum, hippocampus, brainstem, striatum, and cerebellum. Each area of the brain was graded on a 4-point scale: $0=$ no pathology; $1=$ no tissue destruction but minimal inflammation; 2 = early tis- 
sue destruction, demyelination and moderate inflammation; 3 = moderate tissue destruction (neuronal loss, demyelination, parenchymal damage, cell death, neurophagia, neuronal vacuolation); $4=$ necrosis (complete loss of all tissue elements with associated cellular debris). The area with maximum tissue damage was used as the score for that brain region. Each slide was graded by an investigator without knowledge of treatment. For meningeal inflammation, each area was graded as follows: $0=$ no inflammation; $1=$ one cell layer of inflammation; $2=$ two cell layers of inflammation; $3=$ three cell layers of inflammation; $4=$ four or more cell layers of inflammation.

\subsection{Statistical analyses}

T-test or ANOVA on ranks were used for analysis of clinical and pathological scores between treatment groups. Statistical results were considered significant if $p<0.05$.

\subsection{Blinding}

All experiments were carefully blinded such that the investigator performing clinical assessments had no knowledge of the treatments groups. Similarly all pathology slides were coded to remove information about treatment assignments.

\section{Results}

\section{1. rHIgM12 or rHIgM22 did not worsen EAE clinical deficits}

We tested if treatment with $\mathrm{rHIgM} 22$ or rHIgM12 in C57/BL6 mice with MOG-induced EAE affected the clinical deficits. Mice treated with rHIgM22, rHIgM12 or PBS at the onset of the disease ( 15 day postimmunization) had no difference in the clinical scores at day 28 (Fig. 1(A)) or at day 41 (Fig. 1(B)). Subsequently, we evaluated whether treating the mice at different time points of the disease would affect the clinical deficits. Mice were treated during the peak of the inflammatory process (i.e. day 28). Still, there was no difference in the clinical scores between the groups treated with $\mathrm{rHIgM} 22$ or the group treated with rHIgM12. No PBS group was used in this group as previous experiments had shown no difference in clinical deficits (Fig. 1(C)). Finally, we tested whether treatment with three doses of rHIgM22 or rHIgM12 given
Table 1

Spinal cord pathology assessment

\begin{tabular}{|c|c|c|c|c|}
\hline \multirow[t]{2}{*}{ Treatment } & \multicolumn{4}{|c|}{ Pathological score } \\
\hline & $N$ & Demyelination $^{+}$ & $\begin{array}{c}\text { Meningeal } \\
\text { inflammation }\end{array}$ & Experiment \\
\hline rHIgM22 & 7 & $22.5 \pm 4.2^{\mathrm{a}}$ & $22.6 \pm 2.9^{\mathrm{a}}$ & $\# 1$ \\
\hline rHIgM12 & 13 & $22.5 \pm 5.0$ & $21.9 \pm 3.3$ & \\
\hline PBS & 9 & $19.5 \pm 4.9$ & $31.0 \pm 5.7$ & \\
\hline rHIgM22 & 6 & $34.8 \pm 8.8^{\mathrm{b}}$ & $21.3 \pm 5.5^{\mathrm{b}}$ & \#2 \\
\hline rHIgM12 & 7 & $32.3 \pm 8.4$ & $17.2 \pm 3.4$ & \\
\hline rHIgM22 & 8 & $38.0 \pm 9.3^{\mathrm{c}}$ & $11.8 \pm 2.7^{\mathrm{c}}$ & \#3 \\
\hline rHIgM12 & 6 & $36.4 \pm 7.4$ & $12.6 \pm 3.1$ & \\
\hline Anti-CD4 & 9 & $3.4 \pm 3.4$ & $0.8 \pm 0.8$ & \\
\hline
\end{tabular}

${ }^{+}$Demyelination and meningeal inflammation scores are presented as the percent of total spinal cord cross sections graded containing significant white matter lesions or at least one layer of inflammation. a,c ANOVA and t-test $\mathrm{t}^{\mathrm{b}}$ comparisons of demyelination or meningeal inflammation scores for rHIgM12 and rHIgM22 were not significantly different between groups $(P>0.05)$. $\mathrm{N}=$ number of mice per treatment group.

at three subsequent days: one day prior, the same day or day after immunization with MOG peptide could affect the clinical outcome. Anti-CD4 (GK1.5) was used as a positive control as this antibody completely prevents the inflammatory response in EAE. No difference was found between the mice treated with rHIgM22 or rHIgM12 (Fig. 2). Only one mouse out of nine treated with anti-CD4 had mild clinical deficits.

\section{2. rHIgM22 or rHIgM12 did not alter spinal cord pathology in mice with EAE}

We assessed CNS pathology in C57BL6 mice with MOG-induced EAE using a semi-quantitative analysis of spinal cord cross-sections to detect demyelination and meningeal inflammation. There was no reduction of both demyelination and meningeal inflammation in the spinal cord of C57BL6 mice, regardless of the time point of treatment between mice treated with $\mathrm{rHIgM} 22$ or rHIgM12 (Table 1).

\section{3. rHIgM12 decreased inflammation and tissue destruction in brains of mice with MOG-induced EAE}

We assessed brain pathology in C57BL6 mice with MOG-induced EAE treated with rHIgM12, rHIgM22, PBS or antiCD4 antibodies in experiments \#1 (Figs 3(A)-(C)), \# 2 (Figs 3(D)-(E)) and \#3 (Figs 3(F)$(\mathrm{H}))$. After treating mice at the onset of symptoms, there was protection of most of the brain areas of rHIgM12 treated mice (Figs 3(A), (D) and (F)) when compared to other treatment groups such as rHIgM22 
Experiment \#1: rHIgM12

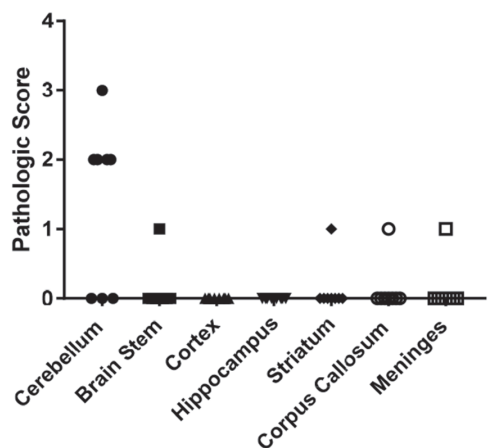

Brain Areas

A

Experiment \#2: rHIgM12

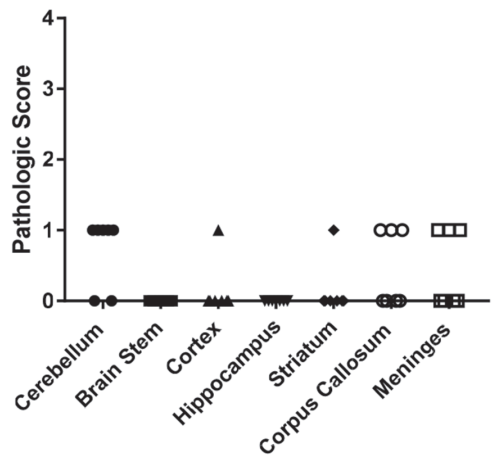

Brain Areas

$\mathrm{D}$

Experiment \#3: rHIgM12

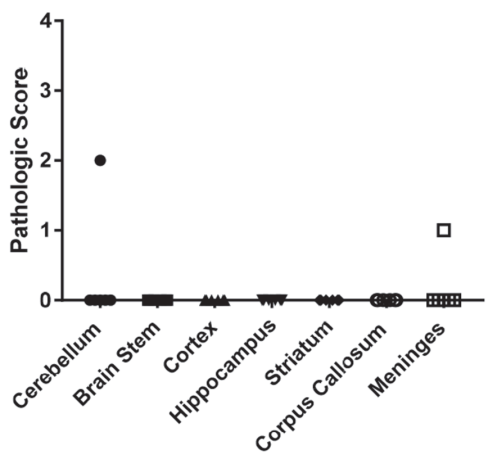

Brain Areas

F
Experiment \#1: rHlgM22

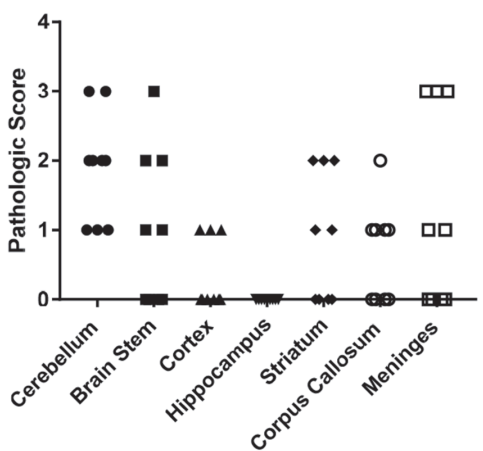

Brain Areas

B

\section{Experiment \#2: rHIgM22}

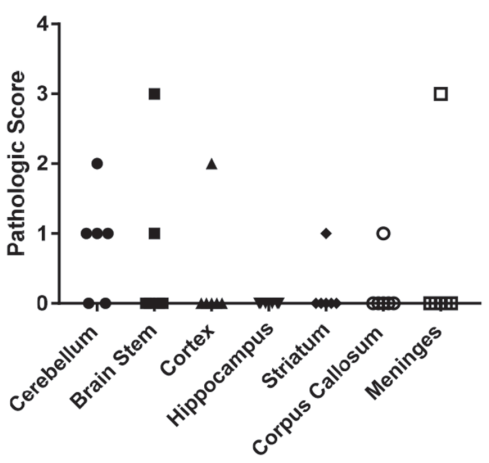

Brain Areas

E

Experiment \#3: rHIgM22

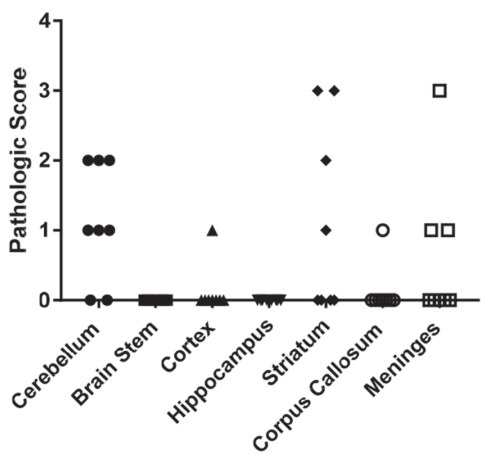

Brain Areas

G
Experiment \#1: PBS

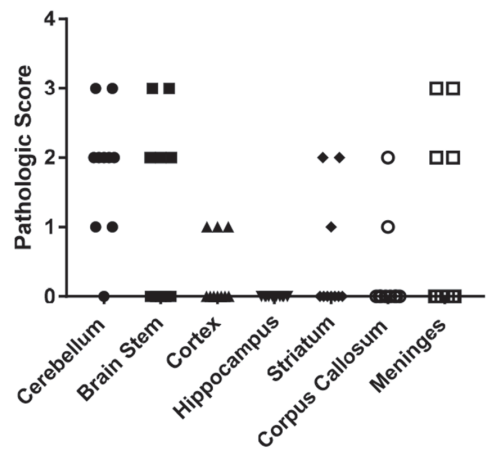

Brain Areas

C
Experiment \#3: anti-CD4

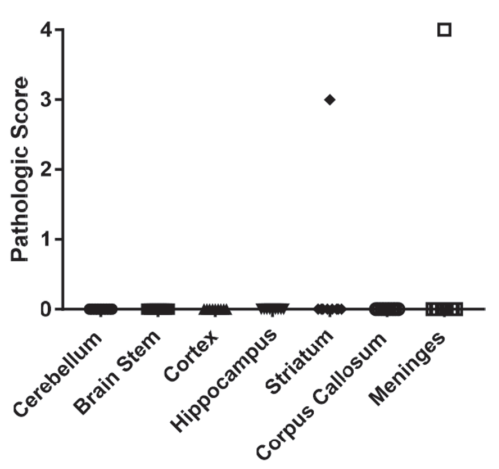

Brain Areas

$\mathrm{H}$

Fig. 3. rHIgM12 given at disease onset (experiment \#1) or at immunization (experiment \#3) reduced brain pathology in mice with EAE. A-C. Experiment \#1: Pathology scores of all brain regions examined at 28 days into disease from mice treated at disease onset with $\mathrm{rHIgM} 12$, rHIgM22 or PBS. Each dot represents one mouse. Comparisons revealed that different brain regions of the rHIgM12 treated group contained substantially less injury than rHIgM22 or PBS treated mice (except for the cerebellum). D-E: Experiment \#2: Pathology scores of all brain regions examined at 41 days into disease from mice treated at disease onset with rHIgM12 or rHIgM22 at the peak of symptoms ( 28 days). Each dot represents one mouse. Comparisons revealed lower pathological scores in most of the brain regions of the rHIgM12 treated mice when compared to rHIgM22 group. F-H: Experiment \#3: Pathology scores of all brain regions examined at 22 days into disease from mice treated at disease onset with rHIgM12, rHIgM22 or anti-CD4. Each dot represents one mouse. Comparisons revealed that different brain regions of the rHIgM12 treated group contained substantially less injury than rHIgM22 and were similar to scores seen in anti-CD4 treated mice. 

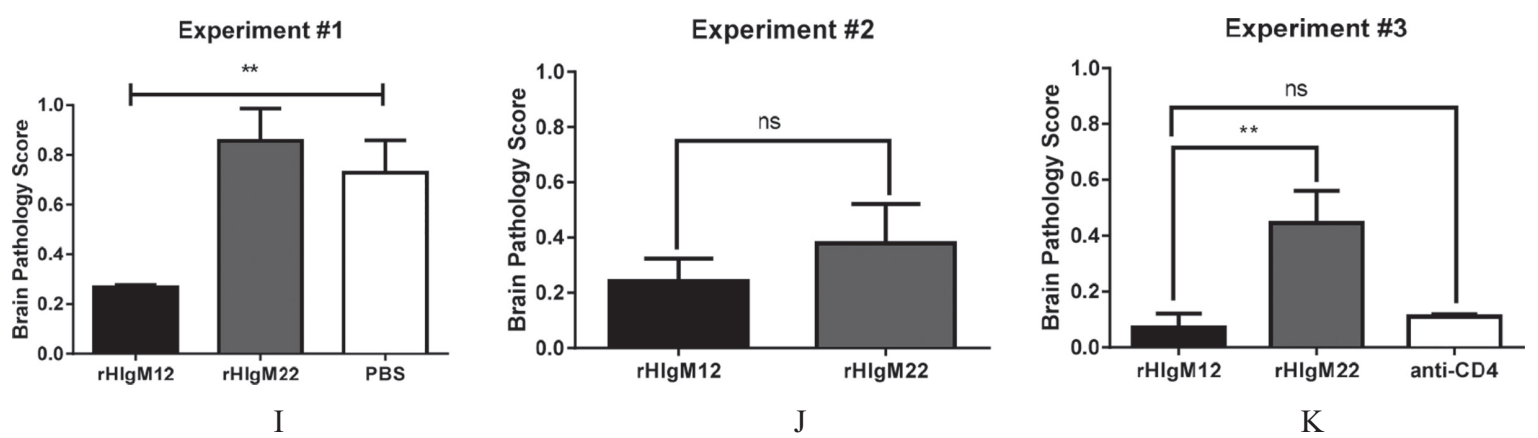

Fig. 3. Continued. I: Experiment \#1: Composite of pathological scores of all brain regions in mice examined at 28 days into disease treated with rHIgM12, rHIgM22 or PBS at day 15 . Comparisons revealed that rHIgM12 treated mice contained substantially less injury than rHIgM22 and PBS treated mice $(P=0.0011)$. J: Composite of pathological scores of all brain regions in mice examined at 28 days into disease treated with rHIgM12 or rHIgM22 Comparisons revealed no difference in the overall pathology score of either group $(P=0.4007)$. K: Composite of pathological scores of all brain regions in mice examined at 28 days into disease treated with rHIgM12, rHIgM22 or anti CD4 antibodies. Comparisons revealed that rHIgM12 treated mice contained substantially less injury than mice treated with $\mathrm{rHIgM} 22(P=0.0082)$ but were not different than mice receiving the CD4 blocking antibody $(P=0.3481)$. Histograms represent the mean pathology score for each group \pm SEM. ANOVA on ranks was used to compare between groups.

or PBS. rHIgM12 treated mice also had a lower composite pathological brain score when compared to rHIgM22 or PBS-treated mice (Fig. 3(I), $p=0.0011$ ). No difference was found in the composite pathological brain score of mice treated with rHIgM12 or rHIgM22 at the peak of the disease (Fig. 3(J), $p=0.4007$ ). Mice treated with rHIgM12 at the time of immunization had less CNS destruction when compared with mice treated with rHIgM22 (Fig. 3(K), $p=0.0082$ ) and the same as the pathology score in mice treated with anti-CD4 (Fig. 3(K), $p=0.3461$ ).

\section{Discussion}

Natural human monoclonal auto-antibodies react to multiple self-antigens with low affinity, thereby potentially acting physiologically. In contrast, conventional auto-antibodies react to a single exogenous antigen with high affinity and thus may be involved in pathologic reactions. One possibility is that natural human antibodies have their therapeutic effect as agonistic antibodies which trigger intracellular signals to induce CNS repair [22].

Our laboratory has developed two unique antibodies, one recombinant 22 which promotes remyelination and recombinant 12 which protects axons from destruction in models of MS and ALS. rHIgM22 is a true human monoclonal antibody that crosses the blood brain barrier [23,24] and targets demyelinating lesions [25]. This antibody increases $\mathrm{Ca}^{2+}$ signaling in oligodendrocytes and astrocytes [26] stimulates proliferation of progenitor oligodendrocytes by reorganizing plasma membranes of oligodendrocyte surfaces and inducing a signaling complex [27]. In contrast, rHIgM12 binds to neurons and protects against axonal injury in chronic TMEV murine model and ALS model [12]. The binding of this antibody to PSA-NCAM and gangliosides of glia and neurons results in neurite extension in vitro and neurite outgrowth [11].

One of the major questions regarding the use of these antibodies is whether they would induce autoimmunity because each of the antibodies is directed against CNS antigens. Therefore, we tested the antibodies in the best established model of CNS autoimmunity, MOG induced EAE.

MOG-induced EAE mice treated with $\mathrm{rHIgM} 22$ or rHIgM12 had no change in the general course of the disease when compared to PBS (Figs 1 and 2). Moreover, no change in meningeal inflammation or demyelination occurred in the spinal cord of mice treated with rHIgM22 or rHIgM12. These results are similar to previous work where rHIgM22 did not influence immunity in the TMEV model [28]. The absence of exacerbation of clinical scores in EAE with either natural human antibody suggests that antibodies would be safe during the acute stages of MS. Thus far, rHIgM22 has been tested in stable MS patients and it has shown no side effects in 55 patients. An active trial is ongoing to assess safety and tolerance in patients with acute attacks (NCT01803867; NCT02398461).

Interestingly, a positive effect was seen in almost all the brain areas of mice treated with $\mathrm{rHIgM} 12$ with the exception of cerebellum. Tissue destruction was less in mice treated with rHIgM12 when compared to mice treated with $\mathrm{rHIgM} 22$ or PBS. Of interest, 
there was a reduction in the meningeal inflammation in brains of mice treated with rHIgM12. This was observed in two separate experiments, thus confirming the findings. Moreover, there was no difference in the brain inflammation of mice treated with $\mathrm{rHIgM} 12$ and antibodies against CD4. In contrast, disease burden in the spinal cord was not altered. This is consistent with previous studies were rHIgM12 had no effect in the inflammation of spinal cord of mice infected with Theiler's virus [10]. The fact that there was a reduction in the brain inflammation in the EAE model and not TMEV infected mice could be explained by the site of the pathologic process. TMEV infected mice have a more localized inflammation during the chronic stage (exclusively in the spinal cord), whereas MOG induced EAE mice have a pathologic disease induced in the periphery. This supports the hypothesis that rHIgM12 may have an immunomodulatory therapeutic effect.

In summary, we have demonstrated that monoclonal natural autoantibodies that have been shown in previous studies to induce either remyelination or neuroprotection do not exacerbate active autoimmune inflammatory diseases. Therefore, these molecules are unlikely to exacerbate neurological deficits during acute phases of MS. Of interest rHIgM12 had an immunemodulatory protective effect in the pathology seen in the brain of MOG-induced EAE mice. A neuroprotective role has also been observed in the spinal cord of chronic TMEV model with axonal injury. Thus, we propose rHIgM22 and rHIgM12 as alternative candidate therapies for human demyelinating diseases such as MS.

\section{Acknowledgments}

This work was supported by a High-Impact Pilot and Feasibility Award (HIPFA) and Novel Methodology Award (NMDA) from the Mayo Clinic Center for Translational Science Activities (CTSA) and Mayo Clinic CTSA grant number UL1 TR000135 from the National Center for Advancing Translational Science (NCATS), a component of the National Institutes of Health (NIH). We also acknowledge with thanks support from the Applebaum, Hilton, Peterson and Sanford Foundations, the Minnesota Partnership Award for Biotechnology and Medical Genomics, the Moon and Marilyn Park Directorship Fund and the McNeilus family. Dr. Lemus is supported by a grant from the Mayo Center for Multiple Sclerosis and Autoimmune Neurology.

\section{References}

[1] A. Denic, S.I. Macura, P. Mishra, J.D. Gamez, M. Rodriguez and I. Pirko, MRI in rodent models of brain disorders, $\mathrm{Neu}$ rotherapeutics $\mathbf{8}(1)$ (2011), 3-18.

[2] A.P. Robinson, C.T. Harp, A. Noronha and S.D. Miller, The experimental autoimmune encephalomyelitis (EAE) model of MS: Utility for understanding disease pathophysiology and treatment, Handb Clin Neurol 122 (2014), 173-189.

[3] A. Ben-Nun, N. Kaushansky, N. Kawakami, G. Krishnamoorthy, K. Berer, R. Liblau et al., From classic to spontaneous and humanized models of multiple sclerosis: Impact on understanding pathogenesis and drug development, J Autoimmun 54 (2014), 33-50.

[4] D. Teitelbaum, A. Meshorer, T. Hirshfeld, R. Arnon and M. Sela, Suppression of experimental allergic encephalomyelitis by a synthetic polypeptide, Eur J Immunol 1(4) (1971), 242248.

[5] Y. Zhang, Y.P. Zhang, B. Pepinsky, G. Huang, L.B. Shields, C.B. Shields et al., Inhibition of LINGO-1 promotes functional recovery after experimental spinal cord demyelination, Exp Neurol 266 (2015), 68-73.

[6] S. Avrameas, Natural autoantibodies: From 'horror autotoxicus' to 'gnothi seauton', Immunology Today 12(5) (1991), $154-159$.

[7] A. Coutinho, M.D. Kazatchkine and S. Avrameas, Natural autoantibodies, Curr Opin Immunol 7(6) (1995), 812-818.

[8] A.E. Warrington, K. Asakura, A.J. Bieber, B. Ciric, V. Van Keulen, S.V. Kaveri et al., Human monoclonal antibodies reactive to oligodendrocytes promote remyelination in a model of multiple sclerosis, Proc Natl Acad Sci U S A 97(12) (2000), 6820-6825.

[9] A.J. Bieber, A. Warrington, K. Asakura, B. Ciric, S.V. Kaveri, L.R. Pease et al., Human antibodies accelerate the rate of remyelination following lysolecithin-induced demyelination in mice, Glia 37(3) (2002), 241-249.

[10] B. Wootla, A. Denic, A.E. Warrington and M. Rodriguez, A monoclonal natural human IgM protects axons in the absence of remyelination, Journal of Neuroinflammation 13(1) (2016), 94.

[11] J. Watzlawik, R.J. Kahoud, S. Ng, M.M. Painter, L.M. Papke, L. Zoecklein et al., Polysialic acid as an antigen for monoclonal antibody HIgM12 to treat multiple sclerosis and other neurodegenerative disorders, J Neurochem 134(5) (2015), 865-878.

[12] X. Xu, A. Denic, L.R. Jordan, N.J. Wittenberg, A.E. Warrington, B. Wootla et al., A natural human IgM that binds to gangliosides is therapeutic in murine models of amyotrophic lateral sclerosis, Dis Model Mech 8(8) (2015), 831-842.

[13] E. Meinl, M. Krumbholz and R. Hohlfeld, B lineage cells in the inflammatory central nervous system environment: Migration, maintenance, local antibody production, and therapeutic modulation, Ann Neurol 59(6) (2006), 880-892.

[14] M. Cruz, T. Olsson, J. Ernerudh, B. Hojeberg and H. Link, Immunoblot detection of oligoclonal anti-myelin basic protein IgG antibodies in cerebrospinal fluid in multiple sclerosis, Neurology 37(9) (1987), 1515-1519.

[15] S. Jarius and B. Wildemann, AQP4 antibodies in neuromyelitis optica: Diagnostic and pathogenetic relevance, Nat Rev Neurol 6(7) (2010), 383-392.

[16] V.A. Lennon, D.M. Wingerchuk, T.J. Kryzer, S.J. Pittock, C.F. Lucchinetti, K. Fujihara et al., A serum autoantibody marker of neuromyelitis optica: Distinction from multiple sclerosis, Lancet 364(9451) (2004), 2106-2112. 
[17] C.P. Genain, B. Cannella, S.L. Hauser and C.S. Raine, Identification of autoantibodies associated with myelin damage in multiple sclerosis, Nat Med 5(2) (1999), 170-175.

[18] T. Berger, P. Rubner, F. Schautzer, R. Egg, H. Ulmer, I. Mayringer et al., Antimyelin antibodies as a predictor of clinically definite multiple sclerosis after a first demyelinating event, $N$ Engl J Med 349(2) (2003), 139-145.

[19] J.B. Sun, T. Olsson, W.Z. Wang, B.G. Xiao, V. Kostulas, S. Fredrikson et al., Autoreactive $\mathrm{T}$ and $\mathrm{B}$ cells responding to myelin proteolipid protein in multiple sclerosis and controls, Eur J Immunol 21(6) (1991), 1461-1468.

[20] Y. Mitsunaga, B. Ciric, V. Van Keulen, A.E. Warrington, M Paz Soldan, A.J. Bieber et al., Direct evidence that a human antibody derived from patient serum can promote myelin repair in a mouse model of chronic-progressive demyelinating disease, Faseb J 16(10) (2002), 1325-1327.

[21] V.P. Van Keulen, B. Ciric, S. Radhakrishnan, K.L. Heckman, Y. Mitsunaga, K. Iijima et al., Immunomodulation using the recombinant monoclonal human B7-DC cross-linking antibody rHIgM12, Clin Exp Immunol 143(2) (2006), 314-321.

[22] S. Gunti, R.J. Messer, C. Xu, M. Yan, W.G. Coleman, Jr., K.E Peterson et al., Stimulation of Toll-Like Receptors profoundly influences the titer of polyreactive antibodies in the circulation, Sci Rep 5 (2015), 15066.

[23] A.D. Eisen, T.J. Button, L. Parry, D. Wang, A. Shah, A.O. Blight et al., Pharmacokinetics of a CNS-penetrating, putative remyelinating human monoclonal antibody, $\mathrm{rHIgM} 22$, in a Phase 1 clinical trial in patients with stable multiple sclerosis (MS). ECTRIMS 2015: ECTRIMS Online Library.
[24] B.M. Greenberg, M. Rodriguez, O.H. Kantarci, D. Arnold, L. Wang, E.J. Carrazana et al., Safety and Tolerability of the Remyelinating Therapeutic Antibody rHIgM22 in Patients with Stable Multiple Sclerosis. Poster $N^{\circ}-$ P4339 | ACO P5130, 67th AAN Annual Meeting | American Academy of Neurology April 18-25, Washington, DC, USA 2015 April 1825;Poster $\mathrm{N}^{\circ}$ - P4.339 | ACO P5130, 67th AAN Annual Meeting | American Academy of Neurology April 18-25, Washington, DC, USA.

[25] I. Pirko, B. Ciric, J. Gamez, A.J. Bieber, A.E. Warrington, A.J. Johnson, D.P. Hanson, L.R. Pease, S.I. Macura and M. Rodriguez, A human antibody that promotes remyelination enters the CNS and decreases lesion load as detected by T2weighted spinal cord MRI in a virus-induced murine model of MS, FASEB J 18(13) (2004), 1577-1579.

[26] M.M. Paz Soldan, A.E. Warrington, A.J. Bieber, B. Ciric, V. Van Keulen, L.R. Pease et al., Remyelination-promoting antibodies activate distinct $\mathrm{Ca} 2+$ influx pathways in astrocytes and oligodendrocytes: Relationship to the mechanism of myelin repair, Mol Cell Neurosci 22(1) (2003), 14-24.

[27] J. Watzlawik, E. Holicky, D.D. Edberg, D.L. Marks, A.E. Warrington, B.R. Wright et al., Human remyelination promoting antibody inhibits apoptotic signaling and differentiation through Lyn kinase in primary rat oligodendrocytes, Glia 58(15) (2010), 1782-1793.

[28] B. Ciric, C.L. Howe, M. Paz Soldan, A.E. Warrington, A.J. Bieber, V. Van Keulen et al., Human monoclonal IgM antibody promotes CNS myelin repair independent of Fc function, Brain Pathol 13(4) (2003), 608-616. 